\title{
La représentation des frontières dans les discours de Marine Le Pen et de Viktor Orbán
}

Renáta Varga

Université de Lille

Cet article propose d'analyser la représentation des frontières dans les discours de deux leaders politiques très médiatisés dans l'espace public national et européen : Marine Le Pen, Présidente du Front National, Députée européenne ${ }^{\mathrm{I}}$ entre $20 \mathrm{I} 4$ et $20 \mathrm{I} 7$ et candidate à l'élection présidentielle de 2017 et Viktor Orbán, Premier ministre hongrois. Interroger le discours politique sous l'angle des frontières offre des clés de compréhension des discours populistes européens actuels car elles cristallisent les tensions liées à la crise des institutions européennes et à la politique de gestion des migrants (Balibar 20I6). Les frontières étant " des marques symboliques, nécessaires aux nations en quête d'un dedans pour interagir avec un dehors » (Foucher 2016:7), elles sont donc largement investies par les leaders politiques. En partant du contexte événementiel de l'arrivée des migrants sur le sol européen et des attaques terroristes en Europe, l'objectif de notre démarche est de montrer les représentations que Marine Le Pen et Viktor Orbán construisent en s'appuyant sur la thématique des frontières, des récits différents qui révèlent des enjeux de légitimation de soi et de prise de pouvoir. Nous souhaitons montrer les similitudes et questionner les différences dans la représentation des frontières de ces deux leaders populistes. Selon l'hypothèse qui sous-tend notre travail, les discours populistes sur les frontières sont multiples et l'investissement de la

I Suite aux élections législatives de 20I7, Marine Le Pen a démissionné de son mandat de Députée européenne le I9 juin 2017 pour occuper son siège de Députée du Pas-de-Calais à l'Assemblée nationale.

Comment citer ce chapitre :

Varga, R. 2019. "La représentation des frontières dans les discours de Marine Le Pen et de Viktor Orbán ». In: Françoise Sullet-Nylander, María Bernal, Christophe Premat \& Malin Roitman (eds.). Political Discourses at the Extremes. Expressions of Populism in Romance-Speaking Countries. Stockholm Studies in Romance Languages. Stockholm: Stockholm University Press, pp. 32I-340. DOI: https://doi.org/Io.I6993/ bax.p. License: CC-BY 
notion de frontière peut être différent en fonction de l'ancrage géo-politique, de la variante du populisme incarné ainsi que des objectifs visés par les leaders populistes.

Cette recherche mobilise l'approche communicationnelle des discours politiques (Charaudeau 2005, 2013, 2016 ; Wodak, 2015). Nous nous intéressons à l'émergence des thématiques en lien avec les frontières et à la mise en cohérence du récit construit à destination des publics cibles. Les discours sont interprétés dans leur contexte socio-politique (Wodak \& Krzyżanowski 20I7 : 472), l'objectif étant de mieux saisir les stratégies des acteurs politiques et les représentations véhiculées. Notre recherche s'appuie sur un éclairage politique permettant de comprendre le populisme comme production de discours et d'actes (Taguieff I984; Enyedi 2016 ; Müller 20I6). Nous envisageons le populisme comme style politique (Taguieff 2007, 20I2 ; Charaudeau 2016 ; Mofitt 20I6) et nous adoptons l'approche sémiologique de Charaudeau qui définit le fonctionnement général du discours populiste comme « une stratégie de conquête ou d'exercice du pouvoir à travers un discours qui reprend la scénographie du discours politique en en radicalisant les imaginaires » (2016 : 37). Pour produire notre analyse, nous nous appuyons sur des travaux des chercheurs en géographie, en sociologie et en philosophie qui questionnent le sens et les fonctions attribuées aux frontières (O’Dowd 2002 ; Groupe Frontière 2004; Ratti \& Schuler 2013 ; Jeandesboz 20I 5 ; Simonneau 20I 5 ; Balibar 20I6 ; Foucher 20I6) et permettent de les interpréter en tant qu'espaces géographiques, culturels et symboliques et en tant qu'imaginaires sociaux.

Notre corpus est constitué de discours oraux et écrits ${ }^{2}$, produits par Marine Le Pen ${ }^{3}$ et Viktor Orbán ${ }^{4}$ entre janvier 20I 5 et avril 2017 dans le cadre national et européen. Il s'agit de différents types d'interventions : des discours officiels, des meetings et des interventions dans les médias : TV ou radio. Nous avons retenu cette période longue car la stratégie de communication de Viktor Orbán autour de la sécurisation

${ }^{2}$ Cette analyse n'intègre pas l'activité des deux leaders sur les nouveaux médias et les réseaux sociaux.

3 Les discours de Marine Le Pen ont été recueillis sur les sites du FN : www.frontnational.com et www.marine20I7.fr. ou directement enregistrés sur les médias nationaux. Notons que le site de campagne Marine 20I7 a été fermé après les élections et le nom du domaine marine20I7.fr a été revendu et transformé en site commercial.

${ }^{4}$ Les discours de Viktor Orbán ont été récupérés dans leur version écrite sur le portail du gouvernement hongrois : http://www.kormany.hu/hu/a-miniszterelnok/beszedek-publikaciok-interjuk. L'ensemble des discours du Premier ministre est mis en ligne en version écrite par ordre anti-chronologique à partir du 6 juin $20 \mathrm{I} 4$. 
des frontières débute en janvier 20I 5 , après l'attaque terroriste contre Charlie Hebdo. Par contre dans l'agenda politique de Marine Le Pen, la thématique des frontières devient centrale pendant la campagne présidentielle française, à partir d'octobre 2016 et durant la campagne officielle en 20I7. Nous avons sélectionné trente discours de chaque leader par rapport à l'intérêt du contenu eu égard à l'objet d'études. Notre méthodologie consistait à établir d'abord une liste de thématiques potentiellement en lien avec la notion de frontière : immigration, migration, politique migratoire de l'UE, menaces associées dans le discours à la migration et à l'immigration, sécurisation du territoire, souveraineté, droit de douanes, contrôles aux frontières, libre circulation des hommes et des marchandises. Nous avons ensuite recherché par une lecture attentive des discours, les extraits pertinents en nous attachant au sens. Notre analyse étant qualitative, nous ne traitons pas la fréquence d'apparition des éléments de contenu. L'objectif est de montrer l'articulation des thématiques abordées dans les discours pour en tirer des différences, ou au contraire des points communs.

Après avoir situé ces deux leaders par rapport à leurs positionnements politiques et les styles populistes incarnés (I), nous montrerons quelles visions des frontières ils développent en s'appuyant sur le contexte événementiel d'attaques terroristes et d'arrivée des migrants (2). Leurs récits sur la nécessaire sécurisation des territoires révèlent deux logiques opposées de la souveraineté nationale (3). Ces visions cachent des ambitions politiques de légitimation de soi et de prise de pouvoir et traduisent la représentation des frontières comme symboles identitaires et comme affirmation d'un pouvoir politique.

\section{Les positionnements politiques et populistes de Marine Le Pen et de Viktor Orbán}

Marine Le Pen et Viktor Orbán se positionnent différemment sur l'échiquier politique. Le Front National est qualifié d'extrême-droite et aucun leader politique en France n'a pris le risque jusqu'en $2017^{5}$ de créer des alliances avec les candidats ou élus FN. Marine Le Pen qui rejette cette étiquette a entrepris depuis son élection à la tête du parti en janvier 20I I,

5 Nicolas Dupont-Aignan, candidat souverainiste ayant obtenu 4,7\% au premier tour de l'élection présidentielle de 2017 s'est allié avec Marine Le Pen et a appelé à voter pour le $\mathrm{FN}$ au $2^{\mathrm{e}}$ tour. 
une opération de " dédiabolisation ${ }^{6}$ (Morel 20I3 3 Charaudeau 2013 ; Dézé 2015 ; Alduy \& Wahnich 20I 5 ; Alduy 20I7) en essayant de gommer les attributs d'extrême-droite ${ }^{7}$, dans une visée d'ascension vers le pouvoir ${ }^{8}$. Selon Charaudeau, ce changement de cap « met le FN dans une situation contradictoire, mais en même temps jette de la poudre aux yeux de ceux qui auraient encore peur de se réclamer de ce parti » (20I3: I93). Alduy et Wahnich pointent cependant le double discours de Marine Le Pen (20I $5: 24 \mathrm{I})$ qui a fait évoluer son langage par rapport à celui de son père (Taguieff I984), mais reprend les idées chères à Jean-Marie Le Pen (Dézé 20I5). Ce jeu illusionniste va même jusqu’à gommer le nom de famille «Le Pen » dans les logos de campagne " Marine 2017 » et « Marine Présidente " pour dissocier la fille de l'image négative du père".

Quant au leader du parti Fidesz-Union Civique, Viktor Orbán affiche un positionnement de droite conservatrice et se réclame des valeurs de la démocratie chrétienne ${ }^{\text {I० }}$ (Korkut 20I2 ; Müller 20I6 : 47 ; Varga $2017 \mathrm{~b})$. Mais " le christianisme qu'il épouse ${ }^{\text {II }}$ est celui qui méprise les valeurs libérales, en particulier l'internationalisme et la compassion pour l'humanité mondiale ${ }^{\mathrm{I}}{ }^{2}$. En effet, le Premier ministre a soutenu

${ }^{6}$ Dézé indique notamment le «travail d'ajustement sémantique » qui a consisté à vouloir « imposer un nouveau registre de discours euphémisé » (2015:34).

7 Marine Le Pen s'est adressée aux représentants de l'organisation patronale MEDEF (Mouvement des entreprises de France) lors de la campagne présidentielle 20I7 : "Arrêtez la caricature, je vous en supplie, cela n'apporte pas grand-chose. Les ogres n'existent pas, le grand méchant loup non plus. Mon élection est une chance, il n'y pas de crainte à avoir. » (Paris, 28.03.2017).

${ }^{8}$ Dézé rappelle que « la stratégie de dédiabolisation de Marine Le Pen n’est nouvelle ni dans son principe, ni dans ses modalités. Sa réactivation témoigne simplement de l'inscription du parti dans une logique électorale de conquête du pouvoir. » (201 $5: 44$ ).

9 Avec l'exclusion le 20 août 2015 de Jean-Marie Le Pen, Président d'honneur et fondateur du parti, le FN semble sur le chemin de la « normalisation » (Alduy 20I7 : I I9). Mais il est difficile d'effacer 40 années de radicalité ; c'est ainsi qu'après avoir mis à distance l'image du père, Marine Le Pen a essayé de se détacher du FN déclarant lors de la campagne : « je ne suis pas la candidate du Front National, je suis la candidate soutenue par le Front National " (Interview, France 2, 25.04.2017).

ro Le Premier ministre se définit comme (bon) chrétien et définit son gouvernement et le parti Fidesz comme "people's party community, based on Christian-democratic foundations » (Budapest, 27.02.2015).

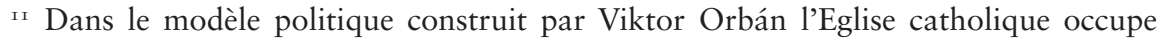
une place importante : en échange des financements, les représentants de l'Eglise soutiennent sans condition l'idéologie du Fidesz. C'est dans cet esprit qu'en septembre 2015 plusieurs dirigeants se sont élevés publiquement contre l'appel du Pape François à accueillir des migrants, rejetant le devoir de compassion (Varga 2OI $7 \mathrm{~b})$.

${ }^{12}$ « I think that the Christian identity reveals to us a completely clear order of 
que « la compassion envers les non-autochtones est une forme de politiquement correct " (Fekete $2016: 4^{2-43}$ ). Ce positionnement conservateur du parti Fidesz n'empêche pas le gouvernement de s'approprier les thématiques fortes du parti d'extrême droite hongrois Jobbik, telle la construction de la clôture de barbelés à la frontière serbo-hongroise, dans l'objectif de conquérir son électorat.

L'autre différence significative entre Marine Le Pen et Viktor Orbán concerne leur rapport au pouvoir. Marine Le Pen représente le populisme d'opposition contrairement à Viktor Orbán qui est l'un des rares leaders européens à disposer des pleins pouvoirs et qui incarne à ce titre le populisme gouvernemental (Wodak 20I 5 ; Müller 20I6). Depuis son accès au pouvoir en 20I0, Orbán a pris un virage "illibéral » (Bozóki 20I 5), mettant fin dans la pratique à la séparation des pouvoirs et bloquant les contrepouvoirs. Avec une majorité de $2 / 3$ au Parlement, le gouvernement d'Orbán a réécrit la Constitution afin de pérenniser la place des populistes à la gouvernance (Müller 20I6: 65). Enfin, il a réussi à mettre les principaux médias sous contrôle ${ }^{\mathrm{I} 3}$ et construit ce que Magyar appelle un État maffieux (20I6), c'est-à-dire « une forme privatisée de l'Etat parasite " (2016:70).

Enfin Marine Le Pen et Viktor Orbán représentent deux versions du populisme, liées aux contextes historiques, sociaux et culturels propres à chaque pays. Taguieff décrit le style politique du FN comme populisme identitaire et national populisme autoritaire (2007, 20I2), mais l'évolution actuelle du discours du FN le place en réalité entre le populisme identitaire et le populisme protestataire. En effet, Marine Le Pen a fait évoluer le style politique du parti depuis 2012 en déplaçant le curseur depuis un positionnement dominant anti-immigrés vers

importance or priority. First of all, we are responsible for our children, then for our parents. This comes before all else. Then come those with whom we live in our village or town. Then comes our country, and then everyone else may come. » (Kötcse, 05.09.2015).

${ }^{13}$ La domestication des médias (Magyar, 2016), c'est-à-dire le rachat des médias par des oligarques proches du gouvernement, concerne autant la presse écrite, les chaînes TV, les fréquences radio que les portails internet.

Vásárhelyi, M. (2014) : "Médiamonoply » [Monopoly des médias], in B. Magyar (ed.) A magyar polip 2, Budapest, Noran Libro : 4I3-434.

Sixx \& alii. (20I6) : "Pár év alatt a Fidesz elfoglalta a magyar sajtót » [En quelques années, le Fidesz s'est approprié la presse hongroise], Index.Hu, 29 novembre 20 I 6.

Gauquelin, B. (20I6) : "En Hongrie, la concentration des médias inquiète, Le principal titre d'opposition a été racheté par une société proche du Premier ministre, Viktor Orbán ", Le Monde, 7 novembre 2016 : I2. 
un positionnement social, anticapitaliste et antisystème ${ }^{\mathrm{I4}}$ (Charaudeau 20I3). Quant au style politique du Fidesz-Union civique et de son leader, il constitue une variante propre aux pays post-communistes d'Europe centrale désignée comme populisme paternaliste : "Paternalist populism rejects the political correctness of the "inorganic » establishment, but considers the people insufficiently mature to participate autonomously in decision-making, and allows the government, elected by the people, to educate and discipline citizenry. " (Enyedi 20I6:2I). Le populisme d'Orbán s'appuie sur le majoritarisme contre les intérêts des minorités, le flottement entre élitisme et antiélitisme, notamment par la construction d'un Etat illibéral, et les interventions de l'Etat pour détruire les institutions de l'élite. Enfin, ce populisme paternaliste met l'accent sur l'intérêt collectif et les obligations sociales et pense la communauté politique à partir d'une "similitude ethnoculturelle ${ }^{15}$ (20I6:2I) et non pas à partir de la notion de citoyenneté.

\section{Les frontières comme moyen de protection du territoire}

La thématique des frontières est récurrente dans la rhétorique du FN et apparaît historiquement surtout en lien avec les questions de l'immigration $^{16}$, l'économie et la souveraineté nationale. A partir de janvier 20 I 5 , le contexte événementiel introduit dans les discours du FN les thématiques des «migrants » arrivés en Europe ${ }^{17}$ et du «terrorisme islamiste » en lien avec les attentats sur le sol français nourrissant ainsi un récit sur la porosité des frontières. Dans le discours de Viktor Orbán, les frontières, en lien avec la migration et l'immigration, constituent une thématique

${ }^{14}$ Ce positionnement est clairement affiché pendant la campagne présidentielle de 20I7. Lors du meeting du 5 février à Lyon, Marine Le Pen a "désigné » les deux « adversaires » de la France : « le mondialisme financier et affairiste dont l'Union européenne, la finance et l'essentiel d'une classe politique domestiquée sont les serviteurs zélés [et] le mondialisme djihadiste qui porte atteinte à nos intérêts vitaux à l'étranger, mais aussi qui s'implante sur notre territoire national ».

${ }^{15} \mathrm{La}$ perte de l'identité est une obsession pour Viktor Orbán qui a évoqué en février 20 I7 la nécessité de sauvegarder "l'homogénéité ethnique et culturelle » de la Hongrie (Budapest, 28.02.2017).

${ }^{16}$ Comme dans les slogans « la France aux Français » et "On est chez nous ».

${ }^{17}$ Alduy a analysé les discours de Marine Le Pen recueillis entre 2014 et août 2016 et note un changement de mots et de ton à l'automne 2015 à propos des migrants, un " réarmement idéologique et sémantique ». En effet, à partir de septembre 2015 , Marine Le Pen parle de la crise des migrants comme d'un « déferlement » ou d'une " invasion migratoire ", qu'elle associe à une " Europe passoire ». Pour elle, les réfugiés sont des "immigrés clandestins" (20I7 : I3 3 ). 
nouvelle qui a surgi dans l'espace politique hongrois en janvier 2015. En perte de popularité fin 20I4, Viktor Orbán a cherché un moyen de se réhabiliter auprès de ses électeurs en se saisissant de l'attaque terroriste à Charlie Hebdo pour associer le multiculturalisme, l'immigration, l'arrivée des migrants sur le sol européen et le terrorisme, dans l'objectif de construire la figure de l'ennemi ${ }^{18}$ (Haraszti 20I 5 ; Varga 20I6). Ces deux leaders se sont donc emparés du contexte événementiel pour développer un discours populiste en associant les frontières à des menaces économiques, sécuritaires et identitaires et en développant ainsi leurs récits autour de la nécessaire protection du territoire.

\subsection{Rétablir et maîtriser les frontières nationales}

Marine Le Pen pointe dans ses discours l'effacement des frontières à l'échelle européenne et s'attaque à la libre circulation des personnes sur le plan sécuritaire. Elle y associe les dangers du terrorisme, bien que les actes auxquels elle fait référence se soient déroulés en France, perpétrés essentiellement par des Français de naissance :

(I) L'Europe sans frontières, c'est l'Europe de tous les trafics. Il faut rétablir des frontières nationales, des frontières qui protègent, des frontières qui dissuadent. L'Europe de Schengen, c'est aussi l'Europe du terrorisme, ce terrorisme dont la menace plane plus que jamais sur la France. (Paris, OI.05.20I6) ${ }^{19}$.

Son discours évolue pendant la période étudiée avec la pression de l'enjeu électoral : de l'expression des doutes (2015) aux certitudes (201620I7), des questions aux affirmations et des suggestions aux exigences. Le discours devient de plus en plus alarmiste et le pathos permet de scénariser des catastrophes contre lesquelles Marine Le Pen dit vouloir protéger les Français. Elle parle ainsi de "pluri-insécurités » qui sont la conséquence de "l'ouverture totale des frontières ». Ce discours de

I8 Orbán mobilise la rhétorique populiste pour déshumaniser et criminaliser les migrants (Varga 20I6) et pour nourrir un récit sur les dangers qu'ils représentent selon lui pour l'économie, la sécurité publique et la culture européenne. L'on observe le glissement de " migrant " à "immigré " "économique ", "illégal ", " clandestin », et potentiellement " terroriste ». Les discours ne posent pas explicitement que les migrants seraient des terroristes, mais la récurrence des références au terrorisme suggère fortement que les attentats sur le sol Européen sont une conséquence directe de la migration (Varga 20I $7 \mathrm{~b}$ ).

19 Pour chaque extrait cité, le lieu ou le cadre, ainsi que la date d'intervention sont indiqués entre parenthèses. 
la peur (Wodak 2015) sert à légitimer sa proposition de rétablir et de maîtriser les frontières :

(2) Maîtriser les frontières, ce n'est pas comme se complaisent à nous caricaturer les adversaires de la France, ériger des barbelés. C'est d'abord avoir des frontières et les maitriser comme tous les pays raisonnables du monde. (Paris, OI.05.20I6).

(3) Je n'ai pas parlé de fermer les frontières, je dis simplement qu'elles doivent exister (...) et permettre de filtrer les terroristes. (Conférence de presse, I6.07.20I6).

La frontière apparaît ici comme barrière protectrice (O'Dowd 2002 ; Groupe Frontière 2004 ; de Saint Victor 2007 ; Ratti \& Schuler 2013) et le contrôle proposé renvoie à une fonction de filtrage (Groupe Frontière 2004 ; Jeandesboz 2015).

Marine Le Pen se place également sur le plan économique pour proposer ce qu'elle appelle le "protectionnisme intelligent ", c'est-à-dire le rétablissement des droits de douane pour des produits importés de certains pays pour lutter contre la " concurrence déloyale ", mais aussi pour s'opposer aux " travailleurs détachés ». Elle apporte cette réponse aux préoccupations des électeurs modestes, confrontés au chômage lié à la désindustrialisation de la France. Les expressions " protection raisonnable » et " protectionnisme intelligent » montrent l'interprétation des frontières sous l'angle de la sécurité de subsistance qu'elles offriraient à la population (Vollmer, 20I7:8).

Marine Le Pen se positionne résolument comme eurosceptique et dénonce le système Schengen duquel elle souhaite sortir la France. Dans sa vision, les frontières administratives opposent un « Nous » Français à un " Eux » Européens (et secondairement extra-Européens) et apparaissent comme une construction symbolique identitaire de protection du territoire et des citoyens. Elle projette ainsi l'image d'une France forte protégée par les frontières :

(4) Les Français ont un choix très simple : soit nous continuons sur la voie d'une dérégulation totale, sans frontières, et sans protection, avec comme conséquences : les délocalisations, la concurrence internationale déloyale, l'immigration de masse, la libre circulation des terroristes. [...] Soit vous choisissez la France, des frontières qui protègent nos emplois, notre pouvoir d'achat, notre sécurité, notre identité nationale. (Déclaration $\mathrm{I}^{\mathrm{er}}$ tour élection présidentielle, 23.04.2017). 


\subsection{Fermer et défendre les frontières de Schengen}

Viktor Orbán quant à lui se positionne sur le plan sécuritaire et militaire et propose la fermeture des frontières. Il investit dans ses discours la frontière serbo-hongroise, lieu symbolique où la frontière nationale et la frontière de l'espace Schengen se superposent. Cela lui permet d'exploiter symboliquement les lisières d'un pays, d'un espace économique (Schengen), mais aussi les délimitations d'une nation et d'une culture (l'Europe). Il réunit ainsi dans ses discours des espaces géographiques, économiques et socio-culturels. Ses discours s'appuient sur la figure de l'ennemi pour alimenter un climat de peur (Haraszti 20I 5), et insistent sur la nécessité de défendre les frontières (Varga 2016, 20I7a) :

(5) I always say this - or try to say this - as gently as I can. I am not a heartless man [...], but the border cannot be protected with flowers and cuddly toys. The border can be protected with fences, police officers, soldiers and weapons. We must protect it. ${ }^{20}$ (Interview, Radio Kossuth, 26.08.2016).

L'action politique principale du gouvernement est la construction d'une clôture de barbelés de $\mathrm{I} 75 \mathrm{~km}$ pour détourner le déplacement des migrants arrivés par la route des Balkans. L'objectif affirmé est de " protéger la nation [...] des invasions extérieures, que ce soit celle des Barbares au sens large, des infiltrations terroristes ou des pressions migratoires " (de Saint Viktor 2007 : I6). Mais cette action est purement politique car " rétablir la visibilité des frontières " ne fait que colmater " l'anxiété culturelle, parfois fantasmée, face aux bruits et aux fureurs du monde » (Foucher $2016: 8$ ). Cette frontière solidifiée apparaît donc dans la communication gouvernementale comme une barrière de protection du territoire (O'Dowd 2002 ; Groupe Frontière 2004 ; Ratti \& Schuler 20I3). Selon Márta Pardavi, Présidente du Hungarian Helsinki Comittee : " cette clôture est présentée comme une vache sacrée, elle assure la protection de notre identité. Il faut donc fêter son existence, la montrer, la photographier; et il existe un éthos selon lequel les policiers et gardes-frontières qui assurent une mission à la frontière font un sacrifice pour la patrie en maintenant l'ennemi à l'extérieur. ${ }^{2 \mathrm{II}}$. Cette clôture est donc investie comme un objet de communication, elle a une fonction

${ }^{20}$ Les extraits longs des discours de Viktor Orbán, sont cités en version anglaise, version certifiée par le cabinet du Premier ministre hongrois et mise en ligne sur : http:// www.kormany.hu/en/the-prime-minister/the-prime-minister-s-speeches.

2i Entretien de recherche réalisé à Budapest, en juillet 2016. 
de "théâtralité politique ( Simonneau 20I5:6I), son existence sert de moyen de légitimation de l'action politique :

(6) « no one is able to enter Hungary [...] This is a slight exaggeration, of course, but in essence it is true that not even a bird can enter the territory of Hungary without being screened. » (Interview, Radio Kossuth, 26.08.20I6).

La frontière apparaît donc dans l'interprétation politique et symbolique de Viktor Orbán, comme une ligne de séparation, avec une fonction de mise à distance (Groupe Frontière, 2004), de l'exclusion de l'autre, de l'étranger (Wodak 20I 5). Or, mettre l'accent sur l'insécurité accroit l'insécurité et la peur alors que les solutions proposées ne peuvent permettre de satisfaire le sentiment d'insécurité des citoyens (Vollmer 2017 : 8), ce qui est propice à alimenter la stratégie populiste de proposer toujours plus d'actions sécuritaires : recruter des gardes-frontières, mobiliser l'armée et construire un second mur. Le récit proposé par Viktor Orbán est donc celui "d'un Etat assiégé, mais protecteur, qui construit à ses confins une barrière pour se protéger des envahisseurs ». Cette dernière fonctionne "comme un outil matériel de réaffirmation de l'ordre souverain " de l'Etat-nation « et de l'efficacité du politique » (Simonneau 20I $5: 6 \mathrm{I}-62$ ).

Mais contrairement à la Présidente du FN, Viktor Orbán est très attaché à l'espace Schengen. Il défend l'ouverture des frontières intérieures car la forte présence des travailleurs hongrois dans les pays de l'UE nécessite la libre circulation des personnes dans cet espace économique. C'est dans cet objectif qu'il entame des négociations avec David Cameroun en janvier 2016 puis avec Theresa May en novembre 2016 pour défendre les intérêts des 95000 Hongrois enregistrés en GrandeBretagne pour qu'ils puissent être traités sans discrimination après le Brexit (Lamour \& Varga 20I7). Aussi, fin 2016, presse-t-il ses homologues, notamment le chancelier autrichien qui ont rétabli provisoirement les frontières intérieures du Schengen (Foucher 2016:39-40) de les démanteler.

Viktor Orbán se plaît à présenter le gouvernement comme le gardien du système Schengen et met en avant le leadership qu'il s'attribue dans l'arène politique européenne pour le travail accompli :

(7) Millions of unidentified and unknown people emerged on the southern borders of the continent and Hungary. In 2015 the Hungarian response was loud and clear: controls, identification, interception and turning back, as required under the Schengen Agreement. (Parlement, 22.02.20I6). 
Il projette ainsi l'image d'une Europe forteresse qui isole l'UE de l'extérieur, mais dont les frontières intérieures restent ouvertes. En fin de compte, il exploite de façon ambiguë la superposition des frontières nationales et des frontières de l'espace Schengen et se situe à l'échelle européenne pour proposer deux interprétations des frontières. Dans la première lecture, politique, la frontière est un marqueur symbolique d'identité (O'Dowd 2002) opposant un « Nous » Hongrois-Européens chrétiens $^{22}$ à un "Eux » migrants-musulmans. Cette lecture projette une guerre de civilisations dans laquelle Viktor Orbán incarne un chef de guerre investi par la mission de défendre la culture européenne. Sa seconde lecture, économique, offre une représentation des frontières (ouvertes à l'intérieur de l'espace Schengen) comme ponts et ressources (O’Dowd 2002), car elles permettent une intégration grâce à la libre circulation (Varga 20I7a).

\section{Opposition à l'UE : moyen d'affirmer la souveraineté des Etats}

La thématique des frontières est mobilisée aussi pour attaquer l'UE et comme moyen d'affirmer la souveraineté des Etats, mais les visions des deux leaders sont opposées. Pour Marine Le Pen, la souveraineté est synonyme de la sortie de l'UE, alors que pour Viktor Orbán, elle signifie l'autonomie des Etat-membres de l'UE et la participation renforcée dans la gouvernance européenne.

\subsection{Sortir la France de l'UE}

Dans son cheminement vers la conquête du pouvoir, Marine Le Pen affiche un positionnement antisystème contre l'UE qu'elle accuse d'être la source de tous les maux. Elle qualifie " ce système européiste tyrannique " (Lyon, 05.02.20I7) d' " escroquerie politique, historique, culturelle, économique et au final humaine» (Milan, 29.01.20I6). Elle souhaite "rendre la France à la France » et sa promesse principale lors de la campagne présidentielle du $20 \mathrm{I} 7$ est le retour à la souveraineté nationale par la sortie de l'UE et de l'euro conditionnée par la tenue

${ }^{22}$ "In other words, what is at stake today is Europe and the European way of life, the survival or extinction of European values and nations - or, to be more precise, their transformation beyond all recognition. [...] we would like Europe to remain the continent of Europeans. » (Băile Tuşnad, Roumanie, 25.07.201 5). 
d'un référendum qui symbolise dans son discours comme dans tous les discours populistes (Taguieff 2007, 20I2 ; Müller 20I6 ; Mofitt 20I6) "l'abolition » de la «barrière ", de la « distance » ou de " toute différence» entre gouvernés et gouvernants» (Taguieff $2007: 285$ ). Cette promesse met en avant à la fois " un imaginaire populaire nourri par les idéaux démocratiques » (op. cit.) et le fantasme d'une démocratie directe où le peuple décide. Le référendum apparaît dans le discours de Marine Le Pen comme un outil de relégitimation ou de délégitimation du leader (élu par le suffrage universel), réinvesti ou désavoué ${ }^{23}$ par la volonté du vrai peuple ${ }^{24}$ (Müller 20I6). Pour le FN, le référendum constitue surtout un outil de communication permettant de surfer sur la vague de la victoire du Brexit, qualifiée d'historique par Marine Le Pen. L'opposition à l'UE mobilisée au service de l'ambition politique de Marine Le Pen apparaît comme un moyen de construction de l'image du leader charismatique (Charaudeau 20I5, 20I6) au niveau national :

(8) Je n'aspire pas à administrer ce qui serait devenu une région, une vague région de l'Union Européenne. Je ne souhaite pas être la Vice-chancelière de Madame Merkel [...]. Je veux être la Présidente de la République française qui respecte à nouveau l'article 5 de la Constitution qui fait du Président de la République le garant de l'indépendance nationale et de l'intégrité du territoire. (Débat télévisé, TFI, 20.03.2017).

En fin de compte, le positionnement antisystème contre l'UE dévoile tous les ressorts du populisme du FN : l'appel au (vrai) peuple, l'abolition des médiations, la proposition d'une solution simple, de " bons choix » qui inscrivent l'action politique dans une temporalité mythique (Taguieff 2007 : 286) permettant d'améliorer "immédiatement " la "situation » des Français, de "leurs enfants » et de «leurs parents » (Débat télévisé, TFI, 20.03.20I7). L'UE apparaît dans le récit construit comme un système " technocratique ", déconnectée des réalités nationales, au service d'un "mondialisme financier ", " contre les intérêts des peuples européens ». Marine Le Pen propose d'endosser le rôle du protecteur

${ }^{23}$ Marine Le Pen a promis de démissionner de sa fonction de Présidente de la République en cas d'échec du référendum (Interview, France 2, 28.03.20I7).

${ }^{24}$ Le vrai peuple est défini par Marine Le Pen comme la "France oubliée ", " qui souffre en silence ", " qui paie sans rien dire ", " cette France sans voix ", " cette France des abandonnés ", " cette France qu'on appelle profonde et que je préfère qualifier profondément patriote » (Strasbourg, Parlement Européen, 03.09.2016). 
du peuple français et de défendre " les quatre souverainetés : monétaire, économique, législative et territoriale » de la nation (Lyon, 05.02.2017), permettant ainsi à la France de "retrouver sa grandeur ». Le retour à la souveraineté apparaît donc dans ses discours comme solution symbolique contre l'effacement des frontières de l'Europe. Dans cette interprétation, le retour aux frontières administratives assure la capacité de contenance de la communauté nationale et permet ainsi de donner corps à la nation ${ }^{25}$, à cette communauté imaginaire (Anderson 2002).

\subsection{La souveraineté de la Hongrie au sein de l'UE}

Contrairement à Marine Le Pen, le positionnement antisystème de Viktor Orbán ne concerne pas l'UE comme construction politique et économique mais le libéralisme comme système de pensée (Varga, 20I7a). Viktor Orbán se situe dans le cadre européen, qu'il souhaite faire évoluer car il considère que "Bruxelles » fait preuve d'ingérence dans les décisions de l'Assemblée nationale hongroise, en outrepassant ses pouvoirs. Il exige donc l'autonomie du Parlement et demande le renforcement du pouvoir des Premiers ministres dans la gouvernance de l'UE. Il se sert de la thématique des migrants et des frontières pour s'opposer sur cette question aux autres dirigeants et pour faire évoluer le fonctionnement de la gouvernance. Ses attaques se concentrent sur la politique migratoire de l'UE car il considère que "les fanatiques bruxellois de l'internationalisme » mettent " en danger l'Europe » (Budapest, I 5.03.20I6). Viktor Orbán mène alors selon ses propres termes «plusieurs batailles » pour la souveraineté de la Hongrie, dont la plus importante est le refus des quotas de relocalisation décidés en septembre 20I5 par l'UE qui prévoient l'accueil en Hongrie de quelques I 200 réfugiés. Le refus de principe de ces quotas est présenté par le gouvernement comme enjeu prioritaire de défense de la souveraineté nationale et instrumentalisé lors d'un référendum organisé le 2 octobre $2016^{26}$. Ce dispositif de démocratie participative est investi par le Premier ministre durant l'année 2016 comme outil politique de légitimation de soi et comme symbole de l'opposition du peuple hongrois

${ }_{25}$ Debray, R. (2010) : Eloge des frontières, Paris, Gallimard : 6I.

${ }^{26}$ La question posée lors du référendum était : «Do you agree that the European Union should have the power to impose the compulsory settlement of non-Hungarian citizens in Hungary without the consent of the National Assembly of Hungary? » 
à l'UE. Viktor Orbán s'appuie sur le résultat du référendum pourtant invalide ${ }^{27}$ pour communiquer sur ce qu'il considère comme une victoire du peuple hongrois le suivant :

(9) " The EU's proposal is that we should let the migrants in, and that it should mandatorily distribute them among the Member States, and that Brussels should decide on this distribution. The Hungarian people have considered this proposal today, and they have rejected it. The Hungarian people have decided that we Hungarians alone may decide on whom we wish to live together with. » (Budapest, déclaration résultats référendum, 02.10.2016).

Le noyau dur de l'électorat du Fidesz ayant répondu en soutien du gouvernement est présenté dans les discours comme l'incarnation du peuple, qui mandate le gouvernement pour prendre les décisions suggérées par la question posée. Ce détournement d'un dispositif de démocratie participative au service de la propagande gouvernementale (Müller 20I6 : I02), pratique régulière depuis la prise du pouvoir en 20I0, montre l'illusion populiste (Taguieff 20I2) incarnée par Orbán, car selon Enyedi, "Fidesz claims to represent the national interest not in constant debats but in its natural way » (2016 : II). Au niveau national, ce référendum sert surtout à re-mobiliser les électeurs du Fidesz et à détourner l'attention des citoyens des problèmes brulants d'économie, de corruption et de favoritisme politique (Magyar 20I6). Au niveau Européen, le débat sur les quotas de relocalisation offre un espace de négociation des rapports de pouvoir que Viktor Orbán espère favorable grâce à la mobilisation du groupe de Visegrád $\left(\mathrm{V}_{4}\right)$. Ainsi, le Premier ministre vise à élargir sa marge de manœuvre politique, tout en restant dans le cadre européen. (Metz 20I7).

En fin de compte, même si Marine Le Pen et Viktor Orbán développent une vision divergente de la souveraineté, leur interprétation des frontières en lien avec cette thématique renvoie à la même fonction d'affirmation et de reconnaissance d'un pouvoir politique (Groupe Frontière 2004).

${ }^{27}$ Le taux de participation de $46,7 \%$ peut être qualifié de très faible et le taux élevé de votes invalides $(6,3 \%)$ montre l'opposition des électeurs au référendum. Ce résultat s'explique par le fait que l'opposition a encouragé les électeurs à s'abstenir ou à exprimer un vote invalide en réponse à une question qui selon eux " n'avait pas de sens ». 


\begin{tabular}{|c|c|c|}
\hline Thèmes & Marine Le Pen & Viktor Orbán \\
\hline \multirow[b]{2}{*}{ 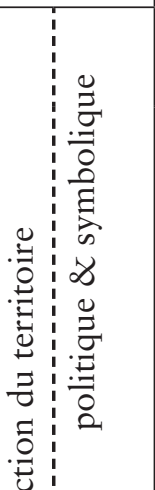 } & Echelle : Etat-nation & $\begin{array}{l}\text { Echelles : Etat-nation / espace } \\
\text { Schengen / UE }\end{array}$ \\
\hline & $\begin{array}{l}\text { - rétablir et maîtriser les } \\
\text { frontières nationales } \\
\text { - filtrer les terroristes } \\
\text { frontières : barrières de } \\
\text { protection } \\
\rightarrow \text { fonction de filtrage }\end{array}$ & $\begin{array}{l}\text { - fermer et défendre les } \\
\text { frontières de Schengen } \\
\text { - protéger la nation } \\
\text { frontières : barrières de } \\
\text { protection } \\
\rightarrow \text { fonction : mise à distance, } \\
\text { exclusion, marqueur } \\
\text { symbolique identitaire }\end{array}$ \\
\hline 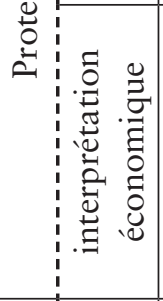 & $\begin{array}{l}\text { - protectionnisme intelli- } \\
\text { gent pour lutter contre la } \\
\text { concurrence déloyale } \\
\text { - } \text { sortir de l'espace Schengen } \\
\rightarrow \text { fonction : sécurité de } \\
\text { subsistance }\end{array}$ & $\begin{array}{l}\text { - ouvrir des frontières à } \\
\text { l'intérieur de l'espace } \\
\text { Schengen } \\
\text { frontières : ponts et ressources } \\
\rightarrow \text { fonction d'intégration }\end{array}$ \\
\hline 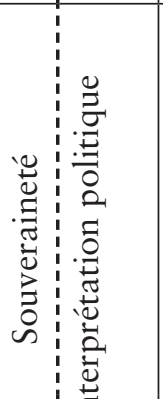 & $\begin{array}{l}\text { - } \text { sortir de l'UE et de l'euro } \\
\text { - } \text { positionnement antisystème } \\
\text { anti-UE } \\
\rightarrow \text { fonction : capacité } \\
\text { de contenance de la } \\
\text { communauté nationale }\end{array}$ & 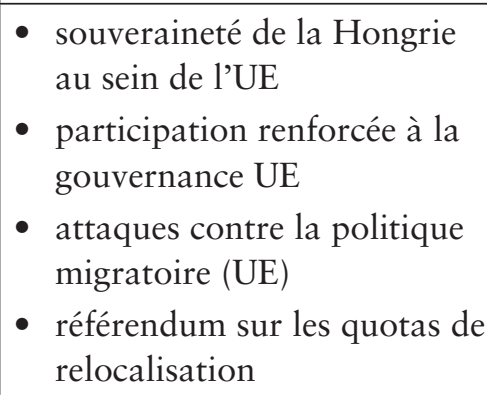 \\
\hline ! & $\begin{array}{l}\rightarrow \text { fonction d'affirmation et de } \mathrm{r} \\
\text { politique }\end{array}$ & econnaissance d'un pouvoir \\
\hline
\end{tabular}

Figure 1 : Thématiques en lien avec les frontières et leurs fonctions. Copyright : Renáta Varga. Licence : CC BY

\section{Conclusion}

Dans les récits élaborés par Marine Le Pen et Viktor Orbán la frontière se trouve au centre du discours populiste, mais son utilisation ne fait sens que par rapport à un symbolisme identitaire situé à plusieurs échelles : par rapport à l'opposition entre les étrangers (Européens/migrants) et le pouvoir de l'étranger (Bruxelles) versus le pouvoir du peuple incarné par les leaders. Les différences dans les représentations analysées ne 
sont pas tant liées aux variantes du populisme incarnées par ces deux leaders qu'au contexte historique des deux pays, à leur position géographique et au poids économique et géopolitique qu'ils représentent en Europe. La synthèse proposée dans la figure I (ci-dessus) montre les thématiques développées par les deux leaders en lien avec les frontières, leur symbolisme, ainsi que les fonctions qui y sont associées. Ce tableau met en évidence que la frontière peut être investie par une interprétation politique (souveraineté et sécurisation du territoire national) ; symbolique (barrière de protection de la nation et d'une culture) ou purement économique (système Schengen).

Dans sa conquête du pouvoir, Marine Le Pen se positionne exclusivement à l'échelle de la nation. Sa lecture politique dessine un récit eurosceptique et souverainiste dans lequel les citoyens européens apparaissent comme sources de menaces économique et sécuritaire. La frontière est marqueur d'identité et représente une barrière administrative, législative et économique. Marine Le Pen propose le repli sur soi à travers sa vision réductrice de la réalité des frontières qui s'oppose à celle du monde globalisé (Ratti \& Schuller 20I3). En effet, selon Balibar, «l'Europe [...] est elle-même une "frontière " complexe : à la fois une et multiple, fixe et mobile, tournée vers l'extérieur et vers l'intérieur. Elle est un Borderland, un «pays-de-frontières » [...] elle forme un espace dans lequel les frontières se démultiplient et se déplacent sans cesse » (2016: I45).

Contrairement à cette interprétation unique, Viktor Orbán fait coexister dans ses discours plusieurs lectures qui se situent à des échelles différentes (Lamour \& Varga 2017 ; Varga 20I7a). Il superpose ainsi les frontières d'un pays, d'un espace économique, mais aussi les délimitations d'une nation et d'une culture. Dans sa lecture symbolique, la frontière en tant que vecteur d'identité apparaît comme rempart pour défendre la civilisation européenne et l'identité chrétienne et révèle une opposition Européens chrétiens versus migrants musulmans. Cet investissement quasi mythique (Varga 20I6) des frontières fait écho à « l'angoisse de la disparition $»^{28}$ du peuple hongrois dont le destin est jalonné de souffrances, de pertes d'autonomie et de territoire, que Viktor Orbán mobilise pour se construire une certaine image. Mais le discours du Premier ministre révèle aussi une lecture économique, plus pragmatique, dans laquelle les frontières ouvertes à l'intérieur de l'espace Schengen sont vecteurs d'intégration et constituent pour les

${ }^{28}$ Pons, F. (201 5 ) : Hongrie : Angoisse de la disparition, Nevicata. 
travailleurs un pont vers les autres pays de l'UE. Cela montre bien la pluralité du discours populiste de ces leaders sur les frontières.

Marine Le Pen et Viktor Orbán mobilisent la notion de frontière dans un enjeu électoraliste de prise de pouvoir. L'ambition de Marine Le Pen est avant tout nationale et le score historique de plus de 10600000 voix, soit $33,90 \%$ des suffrages exprimés, obtenu au $2^{\mathrm{e}}$ tour de l'élection présidentielle le 7 mai 2017 montre que son irrésistible ascension (Alduy et Wahnich 20I $5: 242$ ) vers le pouvoir semble être inéluctablement en marche. Marine Le Pen a incontestablement gagné son pari en réussissant la banalisation du $\mathrm{FN}^{29}$ par l'éloignement de son père, la mise à distance du parti pendant la campagne électorale $20 \mathrm{I} 7$ et sa stratégie entreprise depuis 20 I I de construire une image résolument féminine du leader charismatique (Morel 2013).

Quant à Viktor Orbán, son ambition européenne sert surtout son destin national car son objectif est sa réélection en $20 \mathrm{I} 8$ grâce à un renforcement de sa présence en Europe. Cette stratégie semble efficace car il a réussi à augmenter sa popularité au niveau national ${ }^{30}$ et à accroître sa visibilité dans l'espace médiatique européen au point que certains parlent même de l' «Orbanisation de l'Europe » (Mamadough 20I6), soulignant ainsi son influence sur les discours et les politiques européens. Mais Viktor Orbán joue un jeu d'équilibriste entre les dérives autoritaires de moins en moins cachées et une apparence démocratique de plus en plus fissurée. Ainsi, une loi votée en mars 2017 visant le cadre de fonctionnement de l'université CEU a provoqué d'importantes manifestations à Budapest et l'indignation des intellectuels à travers le monde. Le Premier ministre a été vivement critiqué par la Commission Européenne qui a lancé le 26 avril 2017 une procédure d'infraction contre le gouvernement hongrois. A un an des élections législatives hon-

${ }_{29}$ En 2002 l'arrivée de Jean-Marie Le Pen au $2^{\mathrm{e}}$ tour de l'élection présidentielle française a déclenché une vague de manifestations à travers la France et la classe politique a fait front appelant au vote anti-FN, permettant à Jacques Chirac d'être élu Président de la République avec 82,2 I \% voix. I 5 ans plus tard, l'arrivée de Marine Le Pen au $2^{\mathrm{e}}$ tour de l'élection présidentielle, prédite par les médias et les analystes depuis de longue date, a été accueillie dans une certaine indifférence. Et si certains candidats comme François Fillon (LR) et Benoît Hamon (PS) ont immédiatement appelé à faire barrage au FN, d'autres personnalités politiques ont exprimé à titre individuel leur choix de s'abstenir au $2^{\mathrm{e}}$ tour. Jean-Luc Mélenchon, candidat de "La France insoumise ", qui a farouchement combattu le FN lors de l'élection en 20 I 2 n'a pas donné de consigne claire, indiquant seulement qu'aucun vote ne devait aller au FN.

30 TARKI Social Research Institute, http://www.tarki.hu/hu/news/20I7/kitekint/ 20I70I30_valasztas.html 
groises, Viktor Orbán semblait vouloir tester la force de résistance de la société civile et le seuil de tolérance ${ }^{3 \mathrm{I}}$ de l'UE.

\section{Remerciements}

Je remercie Christian Lamour, chercheur au Luxembourg Institute of SocioEconomic Research pour ses remarques sur cette recherche.

\section{Références bibliographiques}

Alduy, C. (20I7). Ce qu'ils disent vraiment. Les politiques pris aux mots, Paris: Seuil.

Alduy, C. \& Wahnich, S. (2015). Marine Le Pen prise aux mots, décryptage du nouveau discours frontiste. Paris: Seuil.

Anderson, B. (2002) [1983]. L'imaginaire national. Réflexions sur l'origine et l'essor du nationalisme. Paris: Editions La Découverte \& Syros.

Balibar, E. (2016). Europe, crise et fin ? Lormont: Editions Le Bord de l'Eau.

Bozóki, A. (2015). "Broken Democracy; Predatory State, and Nationalist Populism ", in P. Krasztev \& J. Van Til (eds.), The Hungarian patient. Social Opposition to an Illiberal Democracy. Budapest: CEU Press, 3-36.

Charaudeau, P. (2005). Le discours politique. Les masques du pouvoir. Paris: Vuibert.

Charaudeau, P. (2013). La conquête du pouvoir. Opinion, persuasion, valeur. Les discours d'une nouvelle donne politique. Paris: l'Harmattan.

Charaudeau, P. (20I6). " Du discours politique au discours populiste. Le populisme est-il de droite ou de gauche ? ", in J .F. Corcuera \& alii. (eds.), Les discours politiques. Regards croisés. Paris: l'Harmattan, 32-43.

Dézé, A. (2015). "La " dédiabolisation ", une nouvelle stratégie ? ", in Crépon, S., Dézé, A. \& Mayer, N. (eds.), Les faux-semblants du Front National, sociologie d'un parti politique. Paris: Les Presses SciencesPo, 27-50.

Enyedi, Z. (2016). "Paternalist populism and illiberal elitism in Central Europe ». Journal of Political Ideologies 2 I, I, 9-25.

Foucher, M. (2016). Le retour des frontières. Paris: CNRS Editions.

${ }^{31}$ Mudde, C. (2017) : «The EU has tolerated Viktor Orbán for too long. It has to take a stand now ». The Guardian, 3 April 20I7, https://www.theguardian.com/ commentisfree/20I7/apr/o3/eu-tolerated-viktor-orban-hungarian-central-europeanuniversity 
Fekete, L. (2016). "Hungary: Power, punishment and the "Christian-national idea"». Race \& Class 57(4), 39-53.

Groupe Frontière (2004). " La frontière, un objet spatial en mutation ». EspacesTemps.net, Travaux, 04.I0.2004 http://www.espacestemps. net/articles/la-frontiere-un-objet-spatial-en-mutation/

Haraszti, M. (2015). « Behind Viktor Orbán's War on Refugees in Hungary ». New perspectives quarterly 32(4), 37 -40.

Jeandesboz, J. (2015). "Au-delà de Schengen. Frontex et les contrôles aux frontières de l'Europe ", in S. Dullin \& E. Forestier-Peyrat (eds.), Les frontières mondialisées. Paris: PUF, 75-92.

Korkut, U. (20I2). "Work, Home and Order, and Family », in Liberalization Challenges in Hungary. Elitism, Progressivism, and Populism. Palgrave: Macmillan, I68-I77.

Lamour, C. \& Varga, R. (20I7). "The Border as a Resource in Right-wing Populist Discourse: Viktor Orbán and the Diasporas in a Multi-scalar Europe ». Journal of Borderland Studies. Taylor \& Francis (Routledge), I-I6, (First Online 25.II.20I7), DOI: https://doi.org/IO.IO80/08865655.20I7.I402200.

Magyar, B. (2016). Post-Communist Mafia State: The Case of Hungary. Budapest: Noran Libro.

Mamadough, V. (2016). "Beyond the Orbanization of Europe ? Political geographers and global mutual understanding ». IGU Comission on Political Geography $\mathrm{N}^{\circ} 2 \mathrm{I}$ February 20I6, I-3.

Metz, R. (20I7). Határok nélkül ? Orbán Viktor és a migrácios válság [Sans frontières ? Viktor Orbán et la crise migratoire], in A. Körösényi (ed.), Viharban kormányozni. Politikai vezetök válsághelyzetekben [Gouverner dans la tempête. Dirigeants politiques en situation de crise]. Budapest: Magyar Társadalomtudományi Kutatóközpont, 240-264.

Morel, L. (2013). " Les figures contemporaines de leader au prisme de la campagne présidentielle ", in P. Perrineau (ed.), Le vote normal, les élections présidentielle et législatives d'avril-mai-juin 20 I2. Paris: Presses Universitaires Sciences Po, 63-90.

Müller, J.-W. (2016). What is populism? Philadelphia: University of Pennsylvania Press.

O’Dowd,L.(2002). "The Changing Significance of European Borders ». Regional \& Federal Studies I 2, 4, I3-36, DOI: https://doi.org/I0.1080/7 I 4004774.

Ratti, R. \& Schuler, M. (20I3). "Typologie des espaces-frontières à l'heure de la globalisation ". Belgeo [En ligne] I I 20I3, mis en ligne le 3 I octobre 20I3, URL : http://belgeo.revues.org/I0546. 
de Saint Victor, J. (2007). "Le retour des murs: une mondialisation fermée ? ". Cités 3 I, 2007/3. Paris: PUF, I 5-20.

Simonneau, D. (2015). "Militariser la zone frontière. La légitimation des murs, d'Israël à l'Arizona », in S. Dullin \& E. Forestier-Peyrat (eds.), Les frontières mondialisées. Paris: PUF, 59-73.

Taguieff, P.-A. (I984). «La rhétorique du national-populisme [Les règles élémentaires de la propagande xénophobe] ». Mots 9, I I3-I39.

Taguieff, P.-A. (2007) [2002]. L'illusion populiste, Essai sur les démagogies de l'âge démocratique. Paris: Editions Flammarion.

Taguieff, P.-A. (20I2). Le nouveau national-populisme. Paris: CNRS Editions.

Varga, R. (2016). « La construction du mythe de l'ennemi et du héros dans le discours de Viktor Orbán ", in J.F. Corcuera \& alii. (eds.), Les discours politiques. Regards croisés. Paris: l'Harmattan, 3 I4-323.

Varga, R. (2017a). "Construction du leadership national et européen de Viktor Orbán à travers les multiples représentations des frontières ". Management \& Gouvernance $\mathrm{n}^{\circ} \mathrm{I} 8 / 2017$, "La remise en cause des frontières : une nouvelle représentation de la réalité européenne ». Réseau PGV, Université Grenoble-Alpes, 33-40.

Varga, R. (20I7b). "L'identité nationale-chrétienne dans le discours antimigrants de Viktor Orbán ", Journée d'Etudes Le discours politique face aux migrations. Université Montpellier 3, 20-2I octobre 2017.

Vollmer, B. A. (20I7). "Hermeneutical Approach to Eropean Bordering ". Journal of Contemporary Studies 25, I, I-I 5, (First Online 29.02.20 I6).

Wodak, R. (201 5). The politics of fears. What right-wing populist discourses mean. London: Sage.

Wodak, R. \& Krzyżanowski, M. (2017). "Contesting politics \& discourse beyond 'Orbanism' and 'Trumpism' ". Journal of Language and Politics I6, 4, DOI: https://doi.org/IO.I075/jlp.I7042.krz. 